\title{
Los modelos técnico-económicos para análisis de sistemas materiales y su aplicación a la fabricación de piezas de automoción ${ }^{(\bullet)}$
}

\author{
F. Faura \\ $(*)$ \\ Resumen Se discute el potencial de los modelos técnico-económicos, en el marco de los modelos de ingeniería \\ de proceso, explorando para ello el planteamiento de los modelos que se están utilizando actualmen- \\ te. Se ha efectuado una breve revisión de la trayectoria de los mismos, para posteriormente profundi- \\ zar en el modelado de sistemas materiales mediante el análisis de esta metodología. Se han desarro- \\ llado algunos ejemplos indicativos de modelos técnico-económicos que permiten predecir las \\ posibilidades de direccionamiento y desarrollo de los mismos. \\ Palabras clave: Modelos matemáticos. Modelos técnico-económicos. Análisis de sistemas \\ materiales. Procesamiento de materiales.

\section{Technical-economic models for materials systems analysis and their application to automotive parts fabrication}

\begin{abstract}
The potential of the technical-economic models is discussed, in the frame of the models of process engineering exploring the approach of the models that one is utilizing now for it. A brief revision of the trajectory of the same has been effected, for subsequently going deeply into the modeling of material systems through the analysis of this methodology. Some indicative examples of technicaleconomic models have been developed that allow to predict the possibilities of new directions and development from the same.
\end{abstract}

Keywords: Mathematical modeling. Technical-cost modeling. Materials systems analysis. Materials processing.

\section{INTRODUCCIÓN}

La creación y desarrollo de cualquier producto tienen como finalidad fundamental satisfacer una serie de demandas y necesidades existentes en el mercado. Una vez establecidos los requerimientos funcionales del producto se induce un proceso de análisis, a partir del cual se debe ser capaz de establecer los medios adecuados para conseguir el propósito inicial.

Todo sistema productivo puede considerarse como un medio que recibe unos flujos de materia, energía e información (1), de cuya interacción

$(\bullet)$ Trabajo recibido el día 7 de junio de 1996.

(*) Dpto. de Ingeniería de Materiales y Fabricación. E.T.S. de Ingenieros Industriales. Universidad de Murcia. Alfonso XIII, 34. 30203-Cartagena (España). resultará una morfología y unas características finales que deben satisfacer los objetivos funcionales inicialmente planteados. El análisis del sistema puede resultar extremadamente complejo si se consideran todos los factores que afectan a la transformación del material. En una primera aproximación (Fig. 1) se puede apreciar que los requerimientos funcionales del producto condicionan la forma y las características (por tanto el tipo) del material a emplear, los cuales a su vez condicionan la selección del proceso de fabricación a utilizar (2). La toma de decisiones sobre la combinación del tipo de material y ruta o proceso de fabricación a emplear, es, pues, una cuestión compleja a la que se está dedicando un gran esfuerzo de investigación. Muchos de estos esfuerzos están dirigidos a establecer un marco general o una metodología que permita establecer un análisis sistemático y consistente en la toma de decisiones (3). 


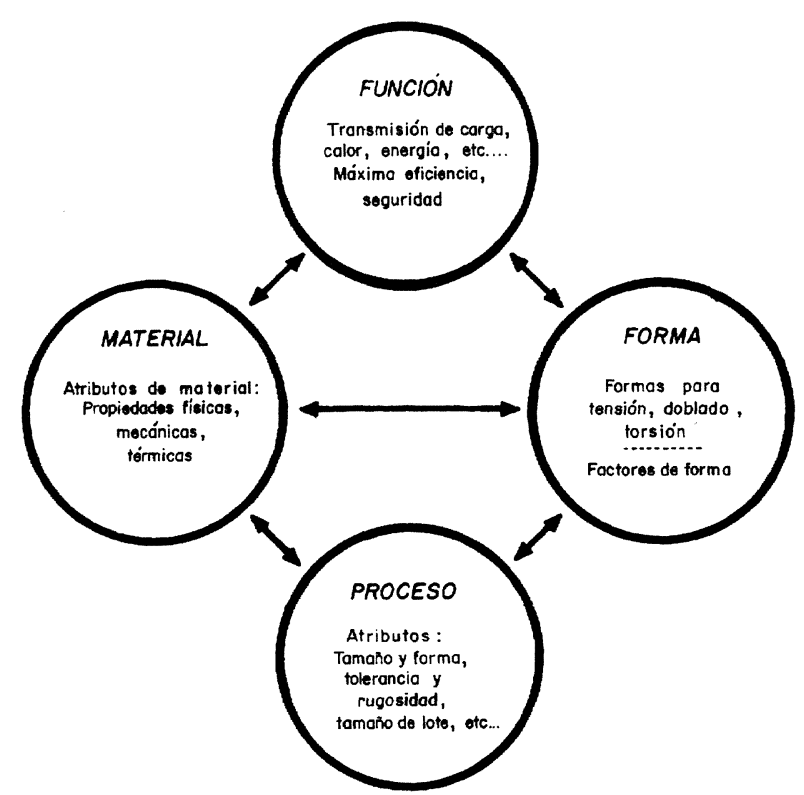

FIG. 1.- Factores implicados en la selección y procesamiento de un material.

FIG. 1.- Factors involved in the materials selection and processing.

Un aspecto fundamental presente en todo este tipo de análisis son las presiones y competitividades propias del mercado. Este aspecto se traduce en una cuestión evidente: no sólo basta con asegurar que el producto satisfaga los requerimientos funcionales, es necesario asegurar, además, que éste sea lo más económico y ecológico posible (4).

En ciertas aplicaciones resulta fundamental el aspecto estético de las piezas fabricadas, así como la flexibilidad del sistema para experimentar adaptaciones rápidas ante modificaciones producidas en el diseño. La flexibilidad ofrecida por ciertas tecnologías de fabricación u otros atributos del proceso y/o producto influyen decisivamente en la toma de decisiones (5).

La emergencia de materiales nuevos y mejores, así como la aparición de nuevas tecnologías de diseño y fabricación, constituyen un entorno dinámico y complejo que plantea la necesidad de un marco formal para poder efectuar proyecciones y/o estimaciones de las consecuencias de decisiones alternativas.

Todos estos factores han propiciado diversos enfoques para asistir a la toma de decisiones. Estos enfoques están convergiendo con el tiempo en el marco de los análisis de sistemas materiales, los cuales, a partir de las técnicas clásicas de análisis de sistemas y de decisión (6 y 7), se dirigen hacia aspectos de trasfondo básicamente económico del ciclo de vida de los materiales. Estas orientaciones alcanzan desde los niveles estratégicos y tácticos (políticas de inversión, impactos de introducción de una tecnología o material), hasta los operativos (costes o beneficios de producción).

Los modelos de análisis que se están desarrollando tratan de aunar los avances producidos en las últimas décadas en los distintos ámbitos de la ingeniería de materiales. Estos modelos buscan la sinergia resultante de la unión de las innovadoras técnicas de modelado matemático, impulsadas por los avances de los denominados materiales de boutique, y los avances producidos en los modelados de control y costes de proceso de los materiales masivos (8).

Diversos planteamientos que muestran este perfil se presentan bajo la etiqueta de Technical Cost Modeling (TCM), constituyendo estos nuevos modelados uno de las materias de investigación de ingeniería de materiales más atractivas.

El objeto del presente artículo es discutir el potencial de los TCM en el marco de los modelos de ingeniería de proceso, explorando para ello el planteamiento de los modelos que se están utilizando actualmente. Se ha efectuado una breve revisión de la trayectoria de los mismos, para posteriormente profundizar en el modelado de sistemas materiales mediante esta metodología. Se han desarrollado algunos ejemplos indicativos de los TCM que permiten predecir las posibilidades de desarrollo de los mismos.

\section{FUNDAMENTOS DE LOS TCM}

Las necesidades de análisis y evaluaciones cuantitativas en la ingeniería de procesado de materiales ha propiciado un fuerte auge de las técnicas de modelización. Los modelos, aunque no satisfacen plenamente la captura de las intrincadas relaciones tecnológicas, económicas y humanas que gobiernan el comportamiento de un sistema material, son las herramientas que presentan más posibilidades a un coste razonable.

Son muchas las posibles orientaciones de los modelos en este entorno. Una relación de los mismos, presentando sus objetivos prioritarios de ámbito económico, podría ser la siguiente:

- Modelos de predicción de producción, consumo y costes.

- Modelos de análisis de la estabilización de mercados.

- Modelos de investigación del impacto de la introducción de un nuevo material o una nueva tecnología en los costes de producción y/o propiedades del material.

- Análisis de la posición competitiva en un cierto escenario de fabricación.

La discusión sobre el ámbito, propósito, prestaciones y limitaciones de las diferentes técnicas de 
modelado es una cuestión abierta. Existe un completo espectro de técnicas de modelado con un frecuente solapamiento entre las diferentes metodologías. En particular, en la modelización de procesos complejos, existe una tendencia de los analistas a tomar prestadas las habilidades de las distintas técnicas, por lo que los nuevos modelos llegan a ser muy difíciles de clasificar.

En general, un TCM se puede definir como una variación de los modelos de proceso convencionales, con particular énfasis en la captura de las implicaciones de los costes, variables de procesos y parámetros económicos.

Desarrollados en el Materials Processing Center, del Massachusetts Institute of Technology (MIT), a finales de la década pasada (9-13), los TCM se presentan como una metodología que considera el material como un flujo que evoluciona económicamente a través del proceso, siendo los factores que afectan a cada fase del proceso de fabricación una función del diseño del producto.

Usando esta metodología, los parámetros críticos se pueden estimar a cada paso del proceso a través de análisis de sensibilidades, pudiendo aplicarse a procesos existentes y a tecnologías nuevas o en desarrollo.

Los TCM permiten, pues, analizar el diseño del sistema material más adecuado para la obtención de un cierto producto. La metodología que subyace en los mismos se puede concretar en los siguientes pasos:

- Identificar los sistemas a analizar, así como las alternativas de diseño para cada sistema.

- Identificar las características fundamentales de cada sistema material, su diseño, fabricación y proceso de montaje.

- Construir los modelos necesarios para estimar las variables de producción, que posteriormente permitan obtener el coste de fabricación de cada alternativa de diseño, desde la materia prima al producto acabado.

- Realizar análisis de sensibilidad para explorar aspectos productivos de los diferentes diseños.

Para una mejor comprensión se puede recurrir a un ejemplo del sector de automoción. Los principales aspectos económicos considerados en la fabricación de elementos de la carrocería de automóviles son el coste de las herramientas, tiempo improductivo, tiempo del ciclo y coste de la materia prima. Tradicionalmente, las carrocerías se fabrican por estampación de chapas de acero. La tecnología de este proceso de fabricación es bien conocida y está ampliamente implantada en la industria del automóvil. Sin embargo, las altas inversiones de capital en equipos y herramientas es una gran desventaja económica en volúmenes de producción pequeños. La mayoría de las tecnologías de compuestos plásticos que pueden emplearse para producir componentes de carrocerías requieren menores niveles de inversiones en equipos y herramientas. No obstante, largos tiempos del ciclo y altos precios de las resinas son fuertes desventajas a superar en el caso de volúmenes de producción elevados.

Este planteamiento constituye la primera fase de la metodología descrita inicialmente, deben identificarse los sistemas materiales mediante la descripción de los componentes considerados y los materiales que pueden emplearse satisfactoriamente. Como puede comprobarse existe una duda importante sobre las ventajas que podrían obtenerse mediante la sustitución del material en este tipo de aplicaciones. Posteriormente, en la segunda fase, se profundiza en los sistemas materiales indicando sus características fundamentales, detallando el diseño físico desarrollado, el proceso de fabricación seleccionado y todas las operaciones de montaje necesarias. Para identificar los principios claves del material y del proceso de fabricación de cada diseño, resulta fundamental tener un alto grado de implicación con las empresas, así como con las personas involucradas en las aplicaciones consideradas. Un importante objetivo consiste en determinar la influencia de las variables del proceso y las características del material sobre el coste final por pieza.

Las propiedades del material determinarán aspectos del producto relacionados con la ingeniería, tales como métodos de unión, tecnología del proceso, etc. Las variables del proceso recogen los principales principios de fabricación que envuelven el entorno productivo. Los factores considerados engloban las fases del proceso y las operaciones secundarias, tasa de producción, piezas desechadas, rendimientos de material, etc. Los requerimientos de mano de obra, equipos y herramientas son elementos clave para la obtención del coste final de producción. En esta fase se requiere una utilización intensiva de los modelos matemáticos disponibles sobre los diversos procesos de conformación de los materiales.

Una vez caracterizado cada uno de los sistemas materiales que pueden emplearse en el procesado de la pieza, puede analizarse el coste de fabricación de cada opción. Los métodos de modelado de costes deben estar basados en un conocimiento sistemático, estimando los factores de costes individuales que constituyen el coste total. Para estimar el coste de cada elemento, se combinan sistemáticamente las relaciones teóricas, los datos empíricos, el análisis de regresión y los criterios relativos a la ingeniería.

Con una detallada descripción de cada alternativa de diseño y los modelos de costes integrados, puede procederse a estimar el coste de fabricación de cualquier componente propuesto. Aunque los 
resultados obtenidos de los modelos serán ampliamente informativos, éstos suministrarán solamente valores correspondientes al escenario específico de fabricación considerado en el modelo. Para dotarlo de una mayor utilidad a la hora de establecer las diferencias entre las alternativas, es necesario tener más información, por ejemplo, la evolución del coste de la pieza para diferentes volúmenes de producción. El coste de fabricación, por tanto, debe estudiarse considerando la estabilidad que presenta ante perturbaciones producidas en las entradas del sistema. Generalmente, estos factores que influyen en el sistema están condicionados fundamentalmente por el entorno de las instalaciones, así como por el propio medio productivo.

\section{CONSIDERACIONES GENERALES SOBRE LA EVOLUCIÓN Y ESTADO ACTUAL DE LOS TCM}

La evolución de los TCM ha estado ligada al desarrollo de los distintos tipos de modelos en el campo de los materiales. Su punto de partida se puede situar cuando se detecta la necesidad de cubrir el vacío que presentan los distintos tipos de modelos del momento. En una primera valoración sobre la filosofía que envuelve a estos planteamientos, se podría decir que los TCM tratan de unir dos disciplinas de modelado, los modelos matemáticos "predictivos" y los modelos de costes "descriptivos".

Con el desarrollo de los ordenadores, los modelos de costes "descriptivos" empezaron a adquirir un cierto rigor científico (14). En vez de explicar el coste con una simple ecuación basada en la mano de obra y el material, se convirtió en relativamente fácil definir ecuaciones de costes en las que figuran los múltiples factores que intervienen e interrelacionándolos. Por ejemplo, las dos ecuaciones siguientes constituyen un enfoque para estimar los costes de la mano de obra directa y de la energía. Además, estas ecuaciones utilizan uno de los enlaces físicos (Duración del ciclo) existente entre estos dos factores del coste.

$$
\begin{gathered}
\text { Costes salariales }=\frac{\begin{array}{c}
\text { Duración del ciclo } \times \text { Salario } \times \\
\text { Núm. ope. }
\end{array}}{\text { Productividad }} \\
\text { Coste energético }=\frac{\begin{array}{c}
\text { Dur. del ciclo } \times \text { Potencia } \times \\
\text { Precio unit. ener. }
\end{array}}{\text { Rendimiento del proceso }}
\end{gathered}
$$

A pesar de que estos modelos de costes representan un gran avance, son todavía inadecuados en muchas situaciones, en particular cuando se trata de tecnologías o procesos nuevos, emergentes, o insu- ficientemente comprendidos, o en tecnologías de fabricación no optimizadas. A pesar de la exactitud informática, estas ecuaciones no aportan bases o guías para estimar las variables técnicas del proceso que ellas incorporan. Se deben especificar la duración del ciclo, potencia, productividad y rendimiento antes de que se puedan estimar los costes de mano de obra y electricidad. En muchas situaciones, estos parámetros técnicos son tan difíciles de estimar como el propio elemento del coste.

En cuanto a los modelos matemáticos "predictivos", su evolución en el campo de los materiales ha sido muy distinta dependiendo de la comunidad a la cual prestaran su servicio. Se pueden clasificar en función de la utilidad del modelo (de investigación o de control), o en función de las técnicas de resolución (analíticas o computacionales); sin embargo, la clasificación más usual es la que establecen los tres grupos siguientes (15): Modelos Teóricos, también denominados Modelos Mecanicistas, los Modelos Semiempíricos y los Modelos Empíricos, Estocásticos'o de Caja Negra. Cuando el ámbito de actuación es exclusivamente un sector productivo, se suelen clasificar como Modelos de Proceso y Producto.

Un análisis de las prestaciones y utilización de los mismos lleva a la apreciación de que los tradicionales modelos de procesos físicos fueron desarrollados, en gran parte, al margen del sector productivo al que han servido posteriormente, mientras que los modelos de costes tradicionales evolucionaron a partir de la influencia del incremento de complejidad sobre un conjunto de principios contables simplistas. Ambas metodologías han proporcionado a sus respectivas disciplinas un aumento de la capacidad analítica, en algunos casos más allá de las necesidades existentes. Sin embargo, ninguna metodología ha sido particularmente bien adaptada para la tarea de guiar la estrategia tecnológica y el desarrollo económico. La detección de esta deficitaria situación puede considerarse como el punto de partida o la fuerza motriz que ha impulsado el desarrollo de los modelos TCM.

En un sistema material, los algoritmos que describen, por ejemplo, la transferencia de calor y los fenómenos de transporte asociados a un proceso, se usan para predecir valores tales como la duración del ciclo, las necesidades de energía y el consumo de materiales. Por otra parte, estos parámetros están directamente relacionados con el coste de los materiales, con la energía y con el trabajo asociados con el proceso. Por lo tanto, las entradas a estos sistemas pueden incluir, por ejemplo, temperaturas, presiones, longitudes o áreas, mientras que las salidas incluirían precios de equipos, coste de las piezas, etc. Con este nuevo enfoque de modelado se puede estimar el coste de los procesos insuficientemente 
comprendidos y se pueden establecer estrategias para optimizar estos procesos (16).

El sistema presentará una serie de entradas que se identificarán con los parámetros técnicos del proceso, y unas salidas que se corresponderán con las características fundamentales de la pieza procesada. Por ejemplo, en un proceso de fundición, las entradas del sistema podrían ser la presión, temperatura del molde, del metal fundido, etc., mientras que las salidas serían el coste final de la pieza, grado de acabado superficial, exactitud dimensional, etc.

El estudio del sistema supone, por tanto, un detallado conocimiento del proceso de fabricación que conjuga todas sus variantes técnicas con los costes de fabricación, o con otros atributos de la pieza que puedan ser de interés. La complejidad del estudio irá aumentando en función del número de salidas del sistema. La dificultad para obtener estas salidas dependerá, en gran medida, del grado de comprensión del proceso y del software existente. A pesar de los complejos modelos mecánicos desarrollados, la carencia del software necesario en algunos procesos crea un gran obstáculo en la profundización de los sistemas.

Una vez planteado el estudio del sistema, se abre todo un abanico de posibilidades para analizar el mismo. Uno de los aspectos fundamentales a considerar es el coste final de la pieza. Como se ha comentado anteriormente, a partir del estudio de sistemas es posible determinar la variación del coste en función de los parámetros técnicos del proceso. Diversas combinaciones de las entradas del sistema pueden satisfacer los requerimientos técnicos precisos, dando origen a diferentes posibilidades que el analista debe considerar. Aunque todas estas combinaciones de los parámetros del proceso pueden ser técnicamente eficientes, no son catalogadas de la misma forma desde el punto de vista económico. Mediante el análisis del sistema puede determinarse, de entre las diferentes posibilidades técnicas existentes, cuál de ellas es la que resulta económicamente más interesante.

El análisis de un sistema alcanza una de las mayores cotas de interés con la aplicación del análisis de sensibilidades. Este análisis permite investigar la estabilidad de las salidas del sistema ante perturbaciones producidas en las entradas. La realización de un análisis de este tipo es un paso clave en el diseño de un proceso de fabricación, pudiendo considerarse de una importancia similar al proceso de optimización. Determinar la estabilidad de una solución óptima del sistema es fundamental si se considera el hecho de que el problema matemático resuelto en cualquier proceso de optimización es sólo una aproximación al problema real. La solución exacta obtenida y empleada para representar la realidad no es, por lo tanto, una solución exacta al problema real de diseño. General- mente, los parámetros estimados, así como los algoritmos representativos del sistema solamente son capaces de simular la situación real. A partir de estos razonamientos, se hace patente la necesidad de comprobar que, aunque son meras aproximaciones las soluciones obtenidas, no experimentan fluctuaciones bruscas ante variaciones en los parámetros técnicos (entradas del sistema).

\section{ALGUNOS EJEMPLOS ILUSTRATIVOS}

Anteriormente, se ha recurrido a un ejemplo del sector de la automoción, para presentar, de forma simple, la metodología que subyace en estos modelos. En dicho ejemplo, se ha planteado la secuencia de pasos que permitirían analizar el impacto o las consecuencias de las decisiones de sustitución de los materiales sobre el producto, en un sistema determinado. Se plantean ahora dos ejemplos donde se analiza el impacto de las decisiones sobre la utilización de distintas tecnologías de procesamiento. En el primero de ellos se analizan los resultados del modelo y en el segundo el planteamiento del mismo; de esta forma es posible tener una visión más completa de las posibilidades y prestaciones de los TCM.

\subsection{Fabricación de un eje de levas}

Uno de los procesos de fabricación más utilizado en la producción de ejes de levas para motores de automóviles es el moldeo en arena. Para tener un conocimiento de los aspectos productivos del proceso, que permita analizar la rentabilidad del mismo con respecto a otros procesos competidores, es preciso profundizar primero en el sistema de fabricación, analizando todas las fases por las que deberá circular el material hasta conseguir sus características finales.

Otros procesos a considerar en la fabricación de ejes de levas pueden ser: forjado de acero, forjado de acero microaleado, eje ensamblado con levas forjadas y mecanizado total. Las grandes diferencias entre el forjado de un acero normal y de otro microaleado radican en la vida de la matriz y en el coste del material. La presencia de elementos microaleados elimina la necesidad de realizar tratamientos térmicos, lo cual proporciona un ahorro en el coste. Además, la mejor maquinabilidad de los aceros microaleados provoca un aumento de la vida de las herramientas y del rendimiento de producción con respecto al proceso de moldeo en arena.

La fabricación del eje de levas mediante ensamblado consiste en situar las levas forjadas convencionalmente sobre un tubo de acero estirado sin soldadura, y posteriormente expandir el eje tubular por 
presión hidráulica para fijar las levas en su lugar. La fabricación mediante el mecanizado completo del eje se realiza a partir de una preforma de acero con máquinas de control numérico. En comparación con los otros procesos, no precisa prácticamente procesamiento previo al mecanizado. Mientras que las técnicas anteriores, tales como la forja y el moldeo necesitan una secuencia de operaciones de procesamiento del material (incluido el mecanizado), este proceso comprende solamente mecanizado.

La construcción del TCM para este ejemplo se desarrolló en el Materials Processing Center, MIT (17). Los costes de producción y su descomposición por factores se muestran gráficamente en la figura 2 pudiéndose destacar los siguientes aspectos de interés:

- La descomposición muestra claramente las ventajas económicas del proceso de moldeo sobre sus competidores.

- El ahorro de material conseguido al usar un eje de levas ensamblado conlleva una reducción neta del coste del material por pieza, a pesar del alto coste del tubo estirado sin soldadura.

- La contribución del coste de mano de obra en el caso del forjado es mayor que en el proceso de fundición debido a la mayor dificultad de mecanizar el acero.

- El alto coste del forjado puede atribuirse no solamente a la cantidad de mecanización requerida, sino, además, al hecho de que para ello se precisan equipos caros.

- Aunque el mecanizado total del eje elimina operaciones en el procesamiento del material (proceso de forma final), no es competitivo económicamente si se compara con el moldeo o la forja.

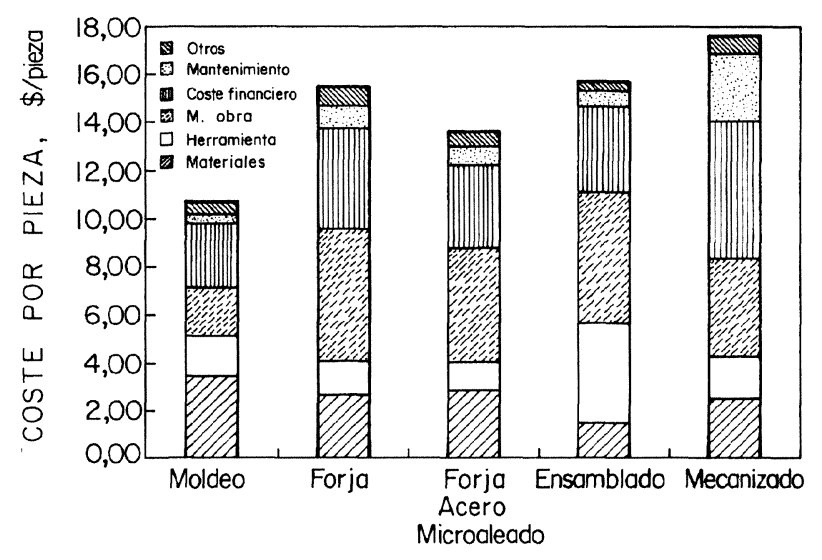

FIG. 2.- Descomposición de costes por factores en la fabricación de ejes de levas.

FIG. 2.- Cost breakup by factor for camshaft manufacturing.

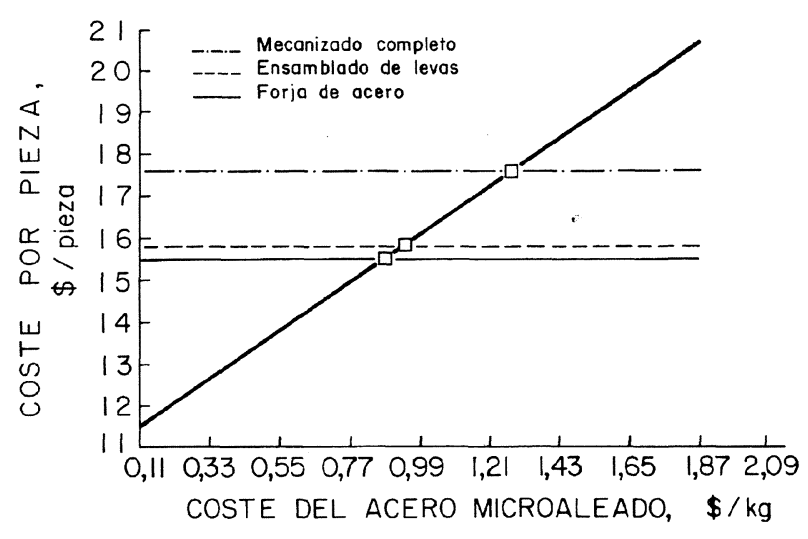

Fig. 3.- Variación del coste total del eje de levas con el coste del acero microaleado en el proceso de forja de acero microaleado.

FIG. 3.- Variation of total cost of camshaft with cost of microalloy steel for microalloy forged cam.

El alto coste de los elementos de microaleación incrementa el coste del material para el eje de levas forjado con acero microaleado con respecto al forjado en acero sin microalear. Debido a que el coste de los elementos de microaleación experimenta importantes fluctuaciones, la variación del coste total con el coste de materia prima podría ser también evaluada. Suponiendo que el coste de las otras materias primas permanezca fijo, la figura 3 muestra el valor que debe alcanzar el coste de acero microaleado para que este proceso sea más caro que el forjado de acero convencional, la fabricación por ensamblaje de piezas o el mecanizado completo del eje.

El moldeo es claramente menos caro que las otras alternativas. El forjado de acero microaleado es más económico que el de acero sin microalear debido a que sus costes de mecanización son menores y no se requieren tratamientos térmicos (temple). La sensibilidad del coste con respecto al volumen de producción aparece representada gráficamente en las figuras 4 y 5.

La sensibilidad del coste de los componentes no mecanizados con respecto al volumen de producción es de gran interés cuando se evalúa un capital elevado o alternativas de fabricación de forma final. En particular, la percepción de la rentabilidad económica puede estar afectada adversamente si el consumidor de una pieza semiacabada no está familiarizado con los requerimientos de mecanizado de las diferentes alternativas y sus respectivos costes. Así, productos semiacabados que en principio pueden presentar un menor coste efectivo, es posible que precisen un mayor coste de mecanizado resultando su coste final más elevado. Por ejemplo, para un volumen de producción de un millón de piezas/año, el moldeo es el proceso de fabricación 


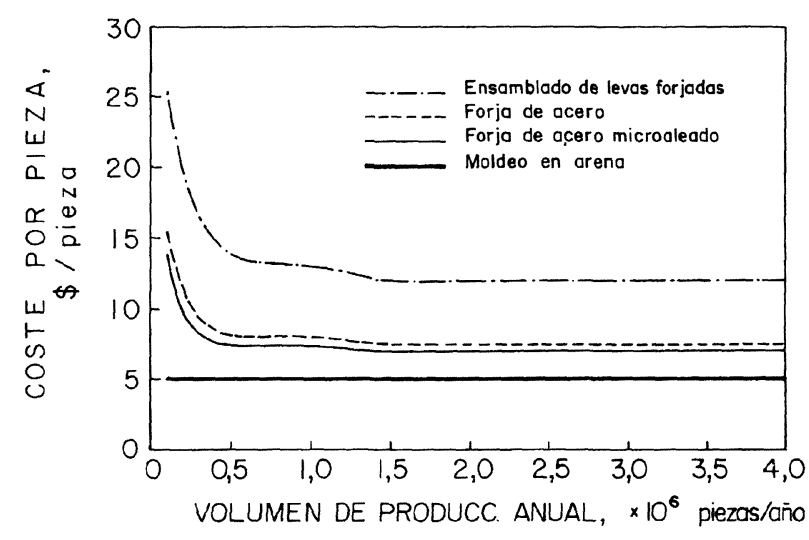

Fig. 4.- Variación del coste del eje de levas con el volumen de producción. Estado semiacabado.

FIG. 4.- Variation of cost with volume in the unfinished state for the case of the camshaft.

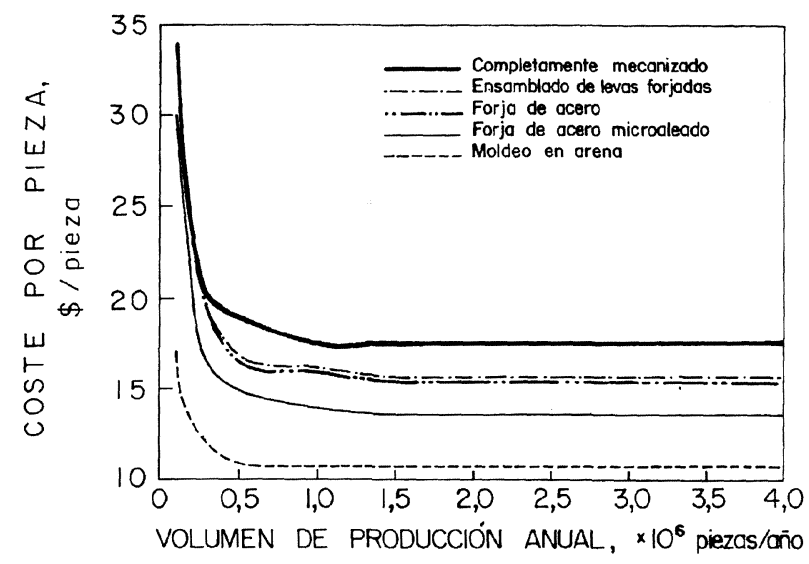

FIG. 5.- Variación del coste con el volumen de producción para el eje de levas completamente acabado.

FIG. 5.-Variation of cost with volume for the fully finished camshaft.

más económico. La figura 4 muestra claramente que el coste de las piezas fundidas sin mecanizar es independiente del volumen de producción. Esto se debe a que el coste de las herramientas es relativamente bajo y los equipos no son delicados; esta suposición se hizo solamente en el caso de fundición debido a que estos procesos, para que resulten rentables, necesitan ser aplicados a grandes volúmenes de producción (excepto en aplicaciones especiales), resultando antieconómico dedicar una instalación a la fabricación de un solo componente. Al incluir la operación de mecanizado el coste de los componentes experimenta una cierta dependencia con el volumen de producción (Fig. 5). Desde un punto de vista estrictamente económico, el moldeo se presenta como el proceso óptimo para fabricar el eje de levas.

\subsection{Fabricación de una llanta de automóvil de aleación ligera}

Las posibilidades de fabricación de llantas de aleaciones ligeras para automóviles son muy variadas. Recientemente, gracias al avance de los modelos matemáticos que permiten simular de forma eficiente el proceso de llenado de moldes y la solidificación del metal vertido en los mismos, están experimentando un gran impulso todos los procesos de moldeo (18). Las razones del incremento de las preferencias de estos procesos frente a otros tradicionales, como los de conformado por deformación plástica, no son únicas, aunque destacan de forma notoria las económicas. Dentro de los procesos de moldeo se dispone de diferentes tecnologías para fabricar estos elementos (19): gravedad, baja presión, alta presión y compresión (squeeze casting), son las más usuales. Las principales características de cada una de estas tecnologías se resumen en la tabla I.

La construcción de un TCM que permita analizar la competitividad entre estas tecnologías (proyecto que está siendo desarrollado en el Materials Processing Center, MIT), puede realizarse a través de un modelo general, aplicable a cada una de ellas, el cual podría seguir un flujo de información como el indicado en la figura 6. El modelo puede estar compuesto por tres módulos diferentes: módulo de entradas y estimación de datos, módulo de cálculos y módulo de resultados.

El módulo de entradas puede presentarse en secciones relativas a: descripción de la pieza, factores de costes externos, condicionantes del proceso y requerimientos de producción. Éstos, junto a los bloques de estimación y base de datos, suministran la información necesaria para el módulo de cálculos. En el caso de procesos con múltiples fases, este bloque se divide en las operaciones individuales que constituyen el proceso completo.

La descripción general del componente especifica el carácter de la pieza que se va a producir. Esta descripción comprende factores tales como dimensiones, peso, material o complejidad. Para procesos que comprenden más de un material, esta sección recoge la descripción total de los materiales, así como las cantidades necesitadas y el orden en que intervienen en el proceso.

Los factores externos son aquellos que definen el entorno económico y de producción en el que se producen las piezas. Estas entradas contienen costes laborales, de mantenimiento, generales, etc. El coste del capital, así como el grado de dedicación del equipo a la producción de la pieza sometida a estudio, también se especifican.

Los condicionantes del proceso se derivan de la elección de la tecnología. Una vez especificada la tecnología, las condiciones del proceso se 
TABLA I.- Comparación de varios procesos de fundición para la producción de componentes de aluminio en automóviles

TABLE I.- Comparison of various casting processes for the production of aluminum auto parts

\begin{tabular}{|l|c|c|c|c|}
\hline Características principales & Gravedad & Baja presión & Alta presión & Compresión \\
\hline Método de llenado & Cuchara & Presión neumática & $\begin{array}{c}\text { Alta velocidad y } \\
\text { Presión hidráulica }\end{array}$ & $\begin{array}{c}\text { Relativa baja veloc. y } \\
\text { Alta presión de inyección }\end{array}$ \\
\hline Tiempo de llenado, $\mathrm{s}$ & $10-30$ & $10-30$ & 1 & 10 \\
\hline Presión de trabajo, atm & 1 & $1+(0,2-0,5)$ & $100-500$ & $500-1.000$ \\
\hline $\begin{array}{l}\text { Tiempo de ciclo, min } \\
\text { (P.e. ruedas alumin.) }\end{array}$ & $5-10$ & $5-10$ & $1-2$ & 2 \\
\hline Temperatura del molde & Alta & Alta & Baja & Baja-Media \\
\hline Precisión dimensional & + & ++ & +++ & +++ \\
\hline Disponibilidad de diseño & +++ & ++ & + & + \\
\hline Productividad & + & ++ & +++ & +++ \\
\hline Calidad & + & ++ & $+/+++++$ & +++ \\
\hline
\end{tabular}

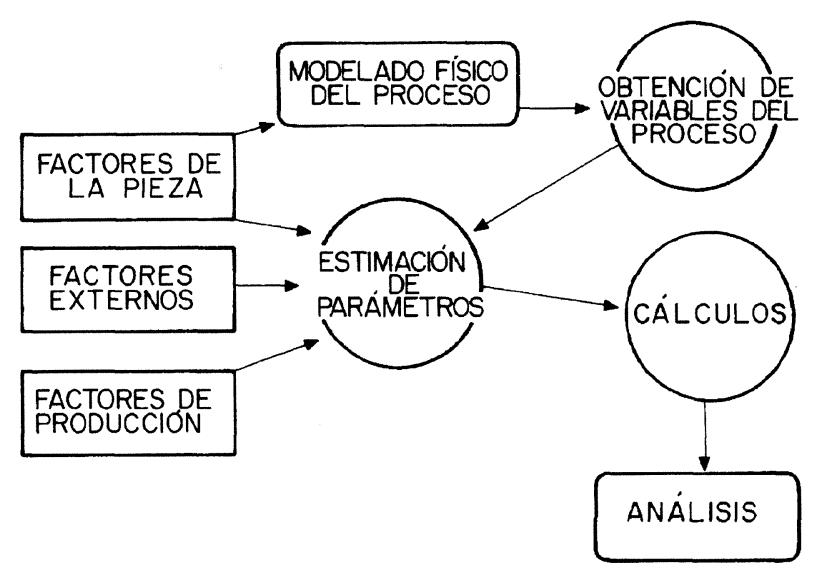

FIG. 6.- Diagrama de flujo de datos en un TCM.

$$
\text { FIG. 6.-Data flowchart in a TCM. }
$$

determinan en cada modelo mediante estudios técnicos que proceden de los módulos de simulación física, o bien a partir de las experiencias adquiridas. En el ejemplo propuesto se puede disponer de distintos modelos matemáticos que permiten, entre otras opciones, simular el proceso de llenado del molde, determinar tiempos de solidificación o predecir la aparición de defectos (poros, rechupes, etc.). A partir de estos análisis, se pueden optimizar parámetros tales como la temperatura y la presión de colada o la duración de ciclo (20).

Los requerimientos de producción definen los objetivos productivos que se pretenden alcanzar (tiempo disponible de producción, volumen de producción, etc.).
En el módulo de estimación, el modelo estima un gran número de los datos necesarios para el módulo de cálculos. Se hacen estimaciones típicas de la capacidad de los equipos, consumo energético, coste de equipos y herramientas, tasas de producción, etc. Las ecuaciones usadas están basadas en expresiones teóricas, análisis de regresión y datos empíricos obtenidos de la industria. En la figura 7 se muestra un ejemplo de ello.

El módulo de cálculo del modelo permite determinar el coste de fabricación por pieza. La efectividad del modelo radica, fundamentalmente, en la capacidad de éste para estimar el mayor número posible de factores, de forma que las entradas necesarias se reduzcan lo máximo posible. El cálculo de costes debe reflejar el incremento del coste del

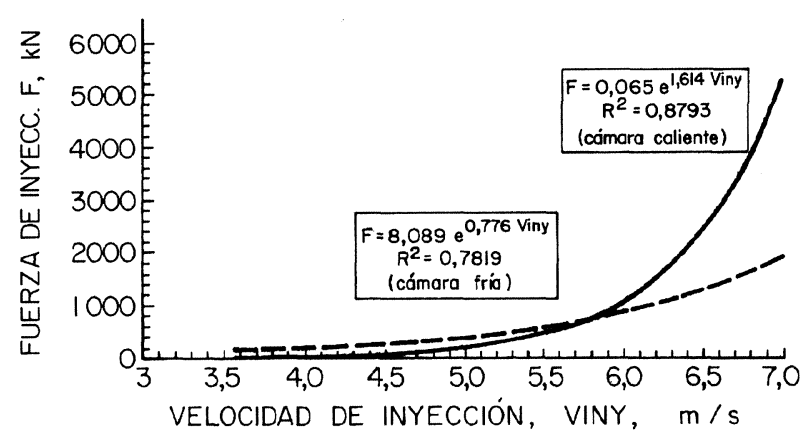

Fig. 7.- Correlación entre la capacidad necesaria de la máquina (fuerza) y velocidad de inyección en el moldeo de aluminio a alta presión.

FIG. 7.- Correlation between power and velocity injection in the aluminum die casting. 
Tabla II.- Descomposición del proceso de fabricación en operaciones individuales y estimación de los rendimientos de cada una de ellas (fundición a contrapresión de aleación de aluminio. Vol. Producción: $10^{6}$ piezas/año)

TABLE II.- Breakup of manufacturing process in individual operations and estimates of the yields of each one of them (counter pressure aluminum alloy die casting. Vol. prod.: $10^{6}$ parts/year)

\begin{tabular}{|l|c|c|c|}
\hline \multicolumn{1}{|c|}{ Operación } & Rendimiento & $\begin{array}{c}\text { Rendimiento } \\
\text { agregado, \% }\end{array}$ & $\begin{array}{c}\text { Núm. } \\
\text { piezas/año }\end{array}$ \\
\hline Fusión y mant. t. & 100 & 86 & 1.165 .369 \\
Moldeo & 96 & 86 & 1.165 .369 \\
Rayos X & 95 & 89 & 1.118 .754 \\
Desbarbado & 99 & 94 & 1.062 .817 \\
Trat. térmico & 100 & 95 & 1.052 .189 \\
Mecanizado & 96 & 95 & 1.052 .189 \\
Acabado & 99 & 99 & 1.010 .101 \\
Expedición & 100 & 100 & 1.000 .000 \\
\hline
\end{tabular}

material al circular a lo largo del proceso productivo. Una vez seleccionada la tecnología de fabricación, se descompone el proceso en las operaciones individuales que forman el mismo, y se procede a la obtención del coste por pieza de cada una de ellas mediante el módulo de estimación de costes por operación. El modelo debe considerar el rendimiento de cada operación teniendo en cuenta el punto del proceso en el que se rechazan las piezas.

A partir de estos rendimientos es posible determinar el número de piezas que es preciso ejecutar en cada operación una vez fijado el volumen de producción. En la tabla II se puede apreciar un ejemplo de estos cálculos para un proceso de fundición a contrapresión.

Los costes parciales por operación se pueden clasificar en dos grupos (fijos y variables). Los costes variables aumentan con el volumen de producción, su aportación a cada pieza permanece constante y están afectados por el rendimiento de producción.

$\mathrm{Al}$ igual que los costes variables, los costes fijos están afectados por un parámetro del proceso. En este caso, se trata del número de líneas de producción. Este afecta a los costes fijos por establecer, para una producción en curso, el tiempo requerido para completar la fase de ejecución.

El efecto del número de líneas sobre el coste de la pieza dependerá de si el equipo usado se dedica únicamente a la producción de ese componente o no. Como se puede observar en la tabla III, un cambio en la duración del ciclo provoca un cambio en la cantidad de líneas necesarias y la inversión de equipos actual será cargada a este componente particularmente para la suposición de equipos no dedicados. En oposición a esto, para el caso de equipos dedicados, la inversión en equipos es invariante debido al número constante de líneas paralelas.

\section{CONSIDERACIONES FINALES}

A partir de las valoraciones y ejemplos desarrollados anteriormente, se desprende una serie de consideraciones que pueden condensarse en las dos reflexiones expuestas a continuación.

Los TCM constituyen una metodología para análisis de sistemas materiales que ha superado ampliamente sus expectativas iniciales. Sus posibilidades actuales han sobrepasado cierto reduccionismo inicial, donde primaban las orientaciones básicamente económicas a niveles operativos (tanto de materiales como del procesamiento de los mismos), acometiendo con más garantías el análisis de niveles superiores que permiten abordar estrategias de desarrollo en procesos insuficientemente comprendidos, emergentes, o en procesos no optimizados. Estos nuevos planteamientos están permitiendo repensar el ciclo de vida del producto, considerando nuevos flujos de información como los de "ecología industrial", y abordar la reingeniería del procesado de materiales.

Aunque el desarrollo de los mismos ha sido espectacular y se han tomado grandes decisiones en diferentes sectores productivos, de indudable éxito, no se puede considerar que esta metodología esté todavía en una fase madura. Las deficiencias más notables se han detectado en la interacción de los modelos matemáticos que simulan el comportamiento físico de los materiales ante un determinado proceso y en los modelos de costes de proceso.

TABLA III.- Influencia del número de líneas de producción sobre el coste de la pieza

TABLE III.- Influence of the number of production lines on the cost of the part

\begin{tabular}{|c|c|}
\hline \multicolumn{2}{|c|}{$\begin{array}{l}\text { SUPOSICIONES: } \\
\text { Volumen de producción }=10.000 \text { piezas/año } \\
\text { Tiempo del ciclo }=0,0166 \mathrm{~h} / \mathrm{pieza} \\
\text { Tiempo total disponible }=3.840 \mathrm{~h} / \text { año } \\
\text { Coste de la máquina }=1.000 .000 \mathrm{PTA}\end{array}$} \\
\hline \multicolumn{2}{|c|}{ Escenario I } \\
\hline Dedicado & No dedicado \\
\hline $\begin{array}{l}\text { Flujos necesarios }=0,04 \\
\text { Flujos paralelos }=1,00 \\
\text { Inversión }=1.000 .000 \text { PTA }\end{array}$ & $\begin{array}{l}\text { Flujos necesarios }=0,04 \\
\text { Flujos paralelos } 0,04 \\
\text { Inversión }=40.000 \mathrm{PTA}\end{array}$ \\
\hline \multicolumn{2}{|c|}{ Escenario II (nuevo t. ciclo $=0,0266 \mathrm{r} / \mathrm{p}$} \\
\hline Dedicado & No dedicado \\
\hline $\begin{array}{l}\text { Flujos necesarios }=0,07 \\
\text { Flujos paralelos }=1,00 \\
\text { Inversión }=1.000 .000 \text { PTA }\end{array}$ & $\begin{array}{l}\text { Flujos necesarios }=0,07 \\
\text { Flujos paralelos }=0,07 \\
\text { Inversión }=70.000 \text { PTA }\end{array}$ \\
\hline
\end{tabular}


Estas dificultades irán desapareciendo conforme se vayan superando las inercias procedentes de los distintos tipos de modelos que se conjugan en los. TCM.

\section{Agradecimiento}

El autor agradece a los Profs. J. Szekely y G. Trapaga sus valiosas sugerencias y las facilidades concedidas para trabajar en su grupo de investigación en el Massachusetts Institute of Technology, MIT (EE.UU.). Asimismo, agradece al Comité Científico de la OTAN el soporte económico concedido para el desarrollo del Proyecto de Investigación en el MIT.

\section{REFERENCIAS}

(1) Alting, L. Manufacturing Engineering Processes. 2 Ed. Marcel Dekker. Nueva York, 1994: 1-10.

(2) Ashby, M.F. Acta Metall. Mater., 39 (6), 1991: 1.0251.039 .

(3) National Research Council. National Material Advisory Board. Comision on Engineering and Technical System. "Analytical Techniques for Studying Substitution among Materials". National Academy Press. Washington, D.C., 1982: 10.

(4) Szekely, J. y Trapaga, G. J. Mat. Res., 10 (9), 1995: 2.178-2.196.

(5) Clark, J.P. y Field III, F.R. Materials Economics, Policy and Management. Ed. M.B. Bever. Pergamon Press. Oxford (R.U.), 1993: 197-204.

(6) De Neufville, R. Applied Systems Analysis. McGrawHill. Singapur, 1990: 297-320.

(7) Goicoechea, A., Hansen, D.R. y Duckstein, L. Multiobjective Decision Analysis with Engineering and Business Applications. John Wiley \& Sons. Nueva York, 1982.
(8) Szekely, J. y Trapaga, G. Modelling. Simul. Mater. Sci. Eng., (2) 1994: 809-828.

(9) Bush, J.V. Technical Cost Modeling of Plastic Fabrication Processes. Ph. D. Thesis. Massachusetts Institute of Technology, 1987.

(10) Hendrichs, N.J. Steel-Plastic Substitutions in Automotive Structural Applications. S.M. Thesis. Massachusetts Institute of Technology, 1989.

(11) Rотн, R. Materials Substitution in Aircraft Gas Turbine Engine Aplication. Ph. D. Thesis. Massachusetts Institute of Technology, 1992.

(12) Neely III, J. The Commercial Potencial for Advanced Ceramics Produced by Low Pressure Injection Molding. S.M. Thesis. Massachusetts Institute of Technology, 1992.

(13) Mangin, C.G. Advanced Engineering Materials for Automotive Engine Components: Cost and Performance Estimations. Ph. D. Thesis. Massachusetts Institute of Technology, 1993.

(14) Poggialy, B. Production Cost Modeling: A Spreadsheet Methodology. S.M. Thesis. Massachusetts Institute of Technology, 1985.

(15) Szekely, J., Evans, J. y Brimacombe, J.K. The Mathematical and Physical Modeling of Primary Metals Processing Operations. John Wiley \& Sons. Nueva York, 1988: 5-8.

(16) Szekely, J. y Trapaga G. Technol. Rev., 98 (1), 1995: 3036.

(17) Nallicheri, N.V. A Technical and Economic Analysis of Alternative Net Shape Processes in Metals Fabrication. $\mathrm{Ph}$. D. Thesis. Massachusetts Institute of Technology, 1989.

(18) KIM, C. y KIM, C-W. Numerical Simulation of Casting Solidification in Automotive Applications. TMS. Pennsylvania (EE.UU.), 1991.

(19) Szekely, J., Trapaga, G. y Saluja, N. An Automated Aluminum Foundry for Production of Automotive Casting. A Feasibility Study. U.S. Trade Development Agency. Washington, DC. Jul., 1993: 4-24.

(20) Otsuka, Y., Trapaga, G. y Szekely, J. Optimal Casting Design by Application of Mold Filling and Solidification Simulation. Light Metals. TMS. 1994: 897-905. 